\title{
REMARKS ON THE CLOSED MODEL CATEGORY STRUCTURE OF SIMPLICIAL SPECTRA
}

\author{
KAJ MALM
}

Introduction. The model category structure of $\mathscr{S} p$, the category of simplicial spectra, has been studied by Hastings in [1] and [2]. His method, in short, is to find analogies in $\mathscr{S}_{p}$ for definitions and theorems presented by Quillen [5] in the case of simplicial sets. As there seem to exist some slips in his proofs (cf. Remarks 2.9 and 2.12), but mainly because his method does not eas.' $y$ allow generalizations, we try here to give a definition of a homotopy structure whin is based on the fact that the spectra can be considered as a special kind of prespectra. In the proofs we use as far as possible only the fact that the components of the prespectra belong to a model category. We shall return to the more general problem of prespectra over model categories in the future.

1. Closed model categories. We recall Quillen's definition of a closed model category [6] and some related concepts.

Definition 1.1. A closed model category is a category $\mathscr{C}$ with three classes of morphisms in $\mathscr{C}$, called fibrations, cofibrations and weak equivalences, satisfying the following axioms:

CM1. $\mathscr{C}$ is closed under finite limits and colimits.

CM2. If $f$ and $g$ are morphisms in $\mathscr{C}$ such that $g f$ is defined and two of $f, g$, and $g f$ are weak equivalences, the third is also a weak equivalence.

CM3. If a morphism $f$ is a retract of a morphism $g$, and if $g$ is a fibration, cofibration, or weak equivalence, then $f$ is also a fibration, cofibration, or weak equivalence, respectively.

CM4. Given the commutative diagram

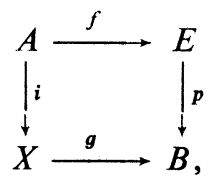


in which $i$ is a cofibration and $p$ is a fibration, there exists a lifting, i.e. a morphism $h: X \rightarrow E$ such that $f=h i$ and $g=p h$ if

(i) $p$ is a weak equivalence, or

(ii) $i$ is a weak equivalence.

CM5. Any morphism $f$ in $\mathscr{C}$ can be factored as $f=p i$, where $p$ is a fibration and $i$ a cofibration and

(i) $p$ is a weak equivalence, or

(ii) $i$ is a weak equivalence.

If the lifting exists in the diagram of CM4, we say that $i$ has the left lifting property with respect to $p$, and $p$ has the right lifting property with respect to $i$. More generally, provided $\mathscr{D}$ is any class of morphisms in $\mathscr{C}$, we say that $i$ has the left lifting property with respect to $\mathscr{D}$ if $i$ has the left lifting property with respect to every morphism in $\mathscr{D}$. Dually, $p$ has the right lifting property with respect to $\mathscr{D}$ if it has the right lifting property with respect to every morphism in $\mathscr{D}$. A morphism which is both a fibration and a weak equivalence is called a trivial fibration, and a trivial cofibration is defined in a corresponding manner.

Lemma 1.2. Suppose that $i: A \rightarrow X$ is a trivial cofibration, $l: B \rightarrow Y$ a weak equivalence and that the inner square in the commutative diagram

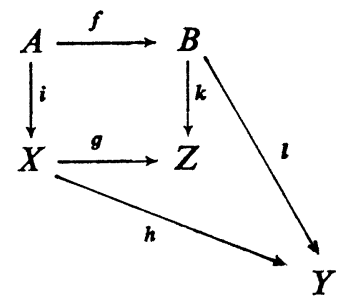

is a pushout. Then the induced morphism $j: Z \rightarrow Y$ is a weak equivalence.

Proof. Factor $k$ as $k_{2} k_{1}$, where $k_{1}: B \rightarrow Z^{\prime}$ is a trivial cofibration and $k_{2}: Z^{\prime} \rightarrow Z$ is a fibration. By CM4 there exists a morphism $m: X \rightarrow Z^{\prime}$ such that $k_{2} m=g$ and $m i=k_{1} f$. Thus there exists a morphism $r: Z \rightarrow Z^{\prime}$ such that $r k=k_{1}$ and $k_{2} r=\mathrm{Id}$. Hence $k$ is a retract of the weak equivalence $k_{1}$ and by CM3 $k$ is a weak equivalence, too. Then by $\mathrm{CM} 2 j$ is a weak equivalence.

2. The closed model category structure of $\mathscr{S}_{p}$. We give definitions of cofibrations, fibrations and weak equivalences in $\mathscr{S}_{p}$ in such a manner that the axioms CM1-CM5 are satisfied and the resulting homotopy category $H o \mathscr{S} p$ is the usual one (i.e. the homotopy category of Kan spectra) up to an equivalence. We recall that there is a functor $P s: \mathscr{S}_{p} \rightarrow \mathscr{P}_{S}$ from the category of simplicial spectra to the category $\mathscr{P}_{S}$ of ss-prespectra such that

$$
\text { 2.1. } P s_{k}(X)_{n}=\left\{x \in X \mid \operatorname{deg} x=n-k, d_{0} \ldots d_{n} x=*, d_{j} x=*, j>n\right\} \text {. }
$$


Definition 2.2. The morphism $f: X \rightarrow Y$ in $\mathscr{S}_{p}$ is

(i) a weak equivalence if $P s_{k}(f)$ is a weak equivalence for every integer $k$,

(ii) a fibration if $P s_{k}(f)$ is a fibration for every $k$,

(iii) a cofibration if $P s_{k}(f)$ is a cofibration for every $k$.

Lemma 2.3. In the pushout diagram

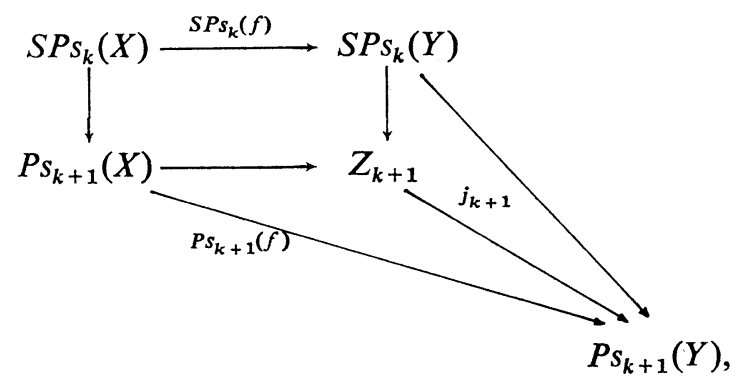

where $f$ is a cofibration and $S$ is the suspension, $j_{k+1}$ is a cofibration for every integer $k$.

Proof. In the category of simplicial sets the cofibrations are precisely the injections.

Remark 2.4. The definition of weak equivalences does not coincide with the classical one given e.g. in [3, Def. 3.3]. However, as we shall see later, the corresponding homotopy categories are equivalent.

Proposition 2.5. The category $\mathscr{S} p$ equipped with the weak equivalences, cofibrations and fibrations of Definition 2.2 is a closed model category.

Proof. As CM1 and CM2 are clear we proceed to CM3. Here it is sufficient to apply $P_{s_{k}}$ to the retraction diagram.

For CM4 suppose we have a commutative diagram

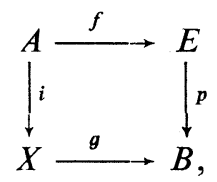

where $i$ is a cofibration and $p$ a trivial fibration. The problem is to find a lifting $X \rightarrow E$. For every $k \geqq 0$ we can find a lifting in the diagram

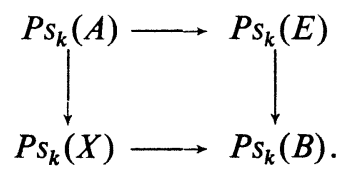


If the morphisms $P s_{k}(X) \rightarrow P s_{k}(E)$ can be chosen in a compatible manner, i.e such that the diagram

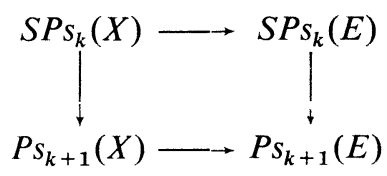

commutes for $k \geqq 0$, they define a morphism $X \rightarrow E$ which solves our problem. Suppose, then, that $h_{k}: P s_{k}(X) \rightarrow P s_{k}(E)$ has been constructed for $k=0, \ldots, n-1$ in such a way that the diagram commutes for these values of $k$. Let $Z_{n}$ be a pushout in the following diagram:

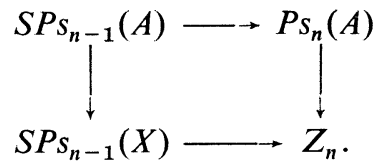

By Lemma 2.3 the induced morphism $Z_{n} \rightarrow P s_{n}(X)$ is then a cofibration. On the other hand, the diagram

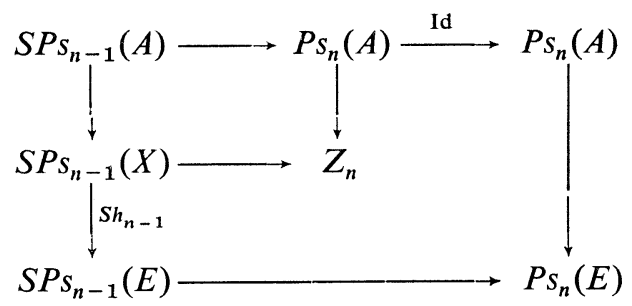

commutes, and so there is a morphism $Z_{n} \rightarrow P s_{n}(E)$ rendering the subdiagrams commutative. Thus we obtain the commutative diagram

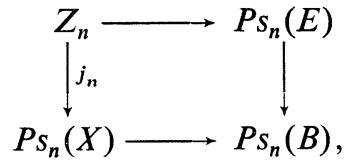

and as $j_{n}$ is a cofibration and the other vertical morphism a trivial fibration, there exists a morphism $h_{n}: P s_{n}(X) \rightarrow P s_{n}(E)$ which is a lifting in the preceding square. Combining these results we see that the restriction of $h_{n}$ to $S P_{s_{n-1}}(X)$ is $h_{n-1}$. The same argument applies with minor modifications if $i$ is supposed to be a trivial cofibration and $p$ a fibration. In fact all the steps prior to the last one remain unaffected; if $i$ is a weak equivalence, then by Lemma $1.2 j_{n}$ is one, too, and the lifting $h_{n}$ exists by CM4 (ii).

To prove CM5 we use the fact that the cofibration - trivial fibration factorization can be performed in a canonical manner in the category of simplicial sets (cf. [5]). We prove only CM5 (i) as the proof of the other half is quite similar. Suppose, then, that $f: X \rightarrow Y$ is a morphism in $\mathscr{S}_{p}$. For every integer $n$ we construct the canonical factorization $p_{n} i_{n}$, where $i_{n}: P S_{n}(X) \rightarrow Z_{n}$ is a cofibration and $p_{n}: Z_{n} \rightarrow$ 
$\rightarrow P s_{n}(Y)$ a trivial fibration. Now the problem is solved if we manage to find a spectrum $Z$ and morphisms $i: X \rightarrow Z, p: Z \rightarrow Y$ such that $P s_{n}(Z)=Z_{n}, P s_{n}(i)=i_{n}$ and $P s_{n}(p)=p_{n}$. But as is easily seen, $w P s_{n+1}(T)=P s_{n}(T)$ for every spectrum $T$, and, conversely, the existence of $Z$ follows if $w Z_{n+1} \cong Z_{n}$ for every integer $n$ (for the definition of $w$ cf. [4, p. 36]). Thus it is enough to prove the following:

Lemma 2.6. Let $X \stackrel{i}{\rightarrow} Z \stackrel{p}{\rightarrow} Y$ be the canonical factorization of a morphism $f: X \rightarrow Y$ in the category of simplicial sets. Then $w(p) w(i): w X \rightarrow w Y$ is isomorphic to the canonical factorization of $w(f)$.

Proof. Recall that $Z$ is defined as $\operatorname{colim}_{n \geqq-1} Z^{n}$, where $Z^{-1}=X, p_{0}=f$ and $Z^{n}, n \geqq 0$, is defined recursively by the pushout diagram

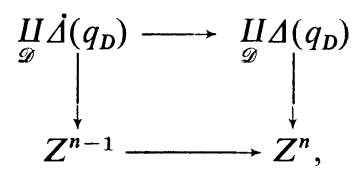

where the index set $\mathscr{D}$ is the set of commutative diagrams

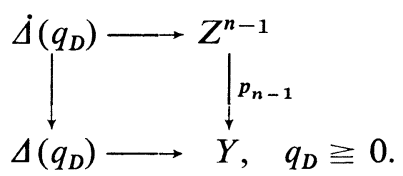

Once $Z^{n}$ is defined we obtain $p_{n}: Z^{n} \rightarrow Y$ in the obvious way; $p$ is then $\operatorname{colim}_{n} p_{n}$ and $i$ is the injection into the colimit. Let $w X \rightarrow V \rightarrow w Y$ be the canonical factorization of $w(f)$; then $V=\operatorname{colim}_{n} V^{n}$, where $V^{n}$ is defined in the same way as $Z^{n}$, with a morphism $q_{n}: V^{n} \rightarrow w Y$ in the same role as $p_{n}$. Finally let $\mathscr{D}^{\prime}$ be the index set in this case. We prove by induction that $w Z^{n}=V^{n}$ and $w\left(p_{n}\right)=q_{n}$ up to an isomorphism, $n \geqq-1$. For $n=-1$ the assertion clearly holds; suppose it holds for $n-1$. Let the diagram $D$,

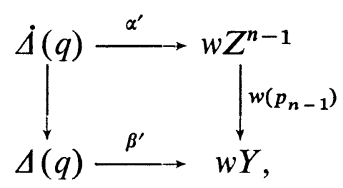

belong to $\mathscr{D}^{\prime}$. If $\beta^{\prime}\left(\delta_{q}\right)=\sigma$, where $\delta_{q}$ is the generator of $\Delta(q)$, then $\sigma$ can be identified with a $q+1$-simplex of $Y$ such that $d_{q+1}(\sigma)=*$ and $d_{0} \ldots d_{q} \sigma=*$, where $*$ is the basepoint. In the same way the simplices $\alpha^{\prime}\left(d_{i} \delta_{q}\right)$ can be identified with $q$-simplices of $Z^{n-1}$. Then we can define morphisms $\beta: \Delta(q+1) \rightarrow Y$ and $\alpha: \Delta(q+1) \rightarrow Z^{n-1}$ such that

$$
\begin{gathered}
\beta(\delta)=\sigma \\
\alpha\left(d_{i} \delta\right)=\left\{\begin{array}{cc}
\alpha^{\prime}\left(d_{i} \delta_{q}\right), & 0 \leqq i \leqq q \\
*, & i=q+1,
\end{array}\right.
\end{gathered}
$$


where $\delta$ is the generator of $\Delta(q+1)$. In this way we obtain an element

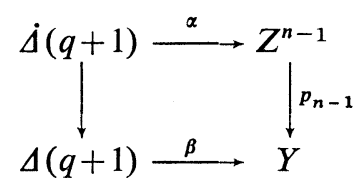

of $\mathscr{D}$, and thus also a commutative diagram

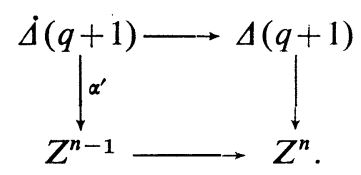

By the definition of $\alpha$ we then obtain a commutative diagram

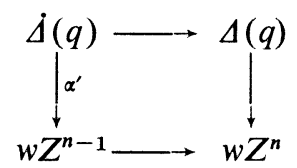

and finally an induced morphism from the pushout $V^{n} \rightarrow w Z^{n}$. Clearly this morphism is monic. To prove it to be epic it is enough to consider a simplex $\sigma \in w Z^{n}$ represented by the generator $\delta_{q} \in \Delta(q), q>0$, where $\Delta(q)$ is associated with the element

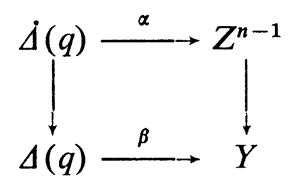

of $\mathscr{D}$. By assumption $\alpha\left(d_{q} \delta_{q}\right)=*$ and $\alpha\left(d_{0} \ldots d_{q-1} \delta_{q}\right)=*$, and thus we obtain an element

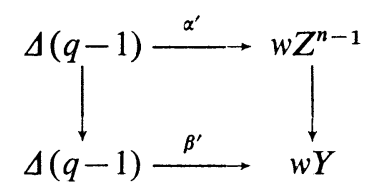

of $\mathscr{D}^{\prime}$. The simplex $\tau \in V^{n}$ represented by $\delta_{q-1}$ clearly maps onto $\sigma$. Finally the relation $w\left(p_{n}\right)=q_{n}$ follows by the universal property of the pushout.

We remark that in the case of fibrations CM3 can be deduced from the fact that the class of fibrations can be characterized as the class of morphisms which have the right lifting property with respect to a suitably chosen class of morphisms. It is well known that such classes are closed with respect to retracts. Recall that $V(k, l)$ is the simplicial subset of $\Delta(k)$ generated by $d_{i} \delta_{k}, i \neq l, 0 \leqq i \leqq k$. To obtain the corresponding inclusion in $\mathscr{S}_{p}$ we employ the functor $\Gamma$ defined in [7]. Recall that $(\Gamma A)_{n}=\operatorname{colim}_{k}\left(S^{k} A\right)_{n+k}$, and the face and degeneracy operations are induced by those of $A$. 
Lemma 2.7. For every spectrum $X$ and every couple $k, t$ of integers,

$$
P S_{k} S^{t}(X)=P s_{k+t}(X)
$$

Proof. An immediate consequence of 2.1 .

Proposition 2.8. A morphism $f: X \rightarrow Y$ in $\mathscr{S}_{p}$ is a fibration if and only if it has the right lifting property with respect to the inclusion $S^{p} \Gamma V(k, l) \rightarrow S^{p} \Gamma \Delta(k)$ for every integer $p, k>0,0 \leqq l \leqq k$.

Proof. Suppose $f$ is a fibration and let the following diagram be commutative:

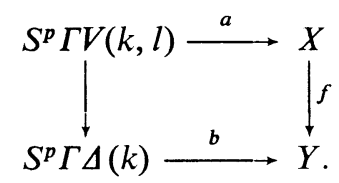

Applying $P s_{-p}$ one obtains, by [4, Lemma 4.7] and Lemma 2.7, the commutative diagram

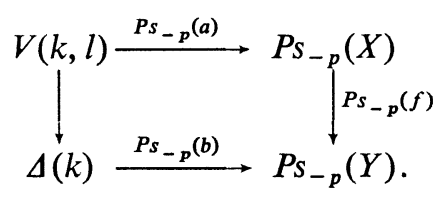

By assumption $P s_{-p}(f)$ is a fibration, and by [5, Def. II. 2.1] this implies the existence of a lifting $\Delta(k) \rightarrow P s_{-p}(X)$. Returning to the category $\mathscr{S}_{p}$ we obtain a commutative diagram

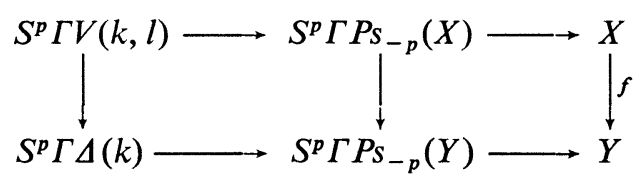

with a lifting $S^{p} \Gamma \Delta(k) \rightarrow S^{p} \Gamma P s_{-p}(X)$; the horizontal morphisms on the right hand are natural injections. As it is easy to see that the horizontal composites are $a$ and $b$, respectively, the desired lifting has been found.

Conversely, suppose $f$ has the right lifting property with respect to every canonical inclusion $S^{p} \Gamma V(k, l) \rightarrow S^{p} \Gamma \Delta(k)$. We claim that $P S_{t}(f)$ is a fibration for every integer $t$. By definition we have to prove that there exists a lifting in the commutative diagram

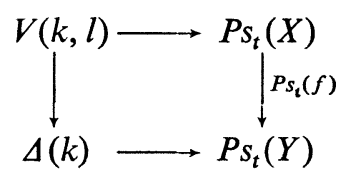


for every integer $t, k>0$ and $0 \leqq l \leqq k$. Applying $\Gamma$ we obtain the diagram

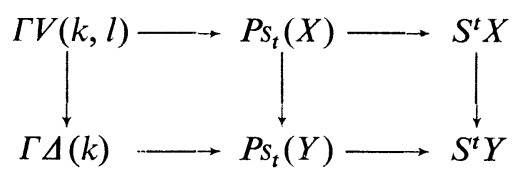

and further, because the suspension is invertible in $\mathscr{S}_{p}$, the diagram

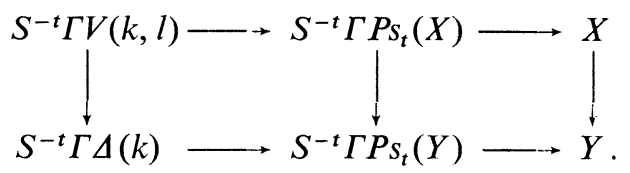

By assumption there is a lifting $S^{-t} \Gamma \Delta(k) \rightarrow X$, and applying $P s_{t}$ we obtain by $[4$, Lemma 4.7] the commutative diagram

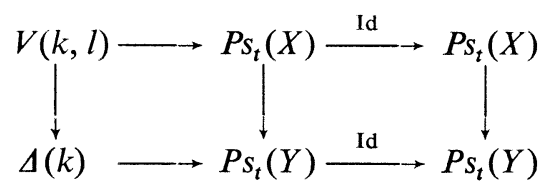

with a lifting $\Delta(k) \rightarrow P s_{t}(X)$.

Remark 2.9. Hastings claims (in [2]) a similar characterization of fibrations (cf. [2, Prop. 5.6 and Prop. 5.10]). In fact he defines objects $\Delta^{k}$ and $V^{k, l}$ in the category of big spectra such that $\Delta^{k}$ is generated by a simplex $\delta_{k}$ in dimension $k$, where $k$ is an integer, and no restrictions are imposed on the faces, i.e. $d_{i} \delta_{k}$ is nondegenerate for all $i \geqq 0$. In the case $f: X \rightarrow *$ this reduces to the assertion that every $P s_{k}(X)$ is a Kan set if and only if every diagram

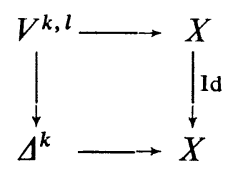

has a lifting. However, this seems to be contradicted by the following:

Example 2.10. Let $X$ be a Kan spectrum (satisfying the local finiteness condition) such that $X_{n}=*$ for $n<0$ and $X_{0}$ contains at least one nondegenerate simplex $\sigma$. Such an $X$ is easy to find, e.g. $X=F \Gamma \dot{\Delta}(1)$ where $F$ is the free group functor defined in [3]. Define a morphism $\Phi: V^{1, l} \rightarrow X$ by setting $\Phi\left(d_{i} \delta_{1}\right)=\sigma$, $i \neq l$. Then $\Phi$ is a simplicial map because $d_{k} \Phi\left(d_{j} \delta_{1}\right)=*$ for every $k \geqq 0, j \neq l$. Suppose $\Phi^{\prime}: \Delta^{k} \rightarrow X$ is a lifting of $\Phi$. Then $\sigma^{\prime}=\Phi^{\prime}\left(\delta_{1}\right)$ is a simplex of $X$ having the property $d_{i} \sigma^{\prime}=\sigma$ for every $i \neq k$, which contradicts the local finiteness condition.

We now turn to the adequacy of the definition of weak equivalences given earlier. As we noted the classical definition of weak equivalences is different from ours, 
namely, weaker. However, the homotopy categories defined by the different notions of w.e. are equivalent. Let $H o \mathscr{S}_{p}$ be the homotopy category (in the sense of Quillen) defined by the model structure of Definition 2.2 .

Proposition 2.11. There exists an equivalence $H o \mathscr{S} p \approx\left(\mathscr{S} p_{E}\right)_{h}$, where $\left(\mathscr{S} p_{E}\right)_{h}$ is the homotopy category of Kan spectra. Moreover Ho $\mathscr{P} \approx \mathscr{S} p\left[\pi^{-1}\right]$, where $\pi$ is the class of classical weak equivalences.

Proof. The latter statement follows from the former by the remark on p. 41 of [4]. To prove the former we recall that an object $X$ of a model category is fibrant if $X \rightarrow *$ is a fibration. By [5, Theorem I. 1.1] there is then an equivalence Ho $\mathscr{S} p \approx H o \mathscr{S}_{p_{f}}$, where $\mathscr{S}_{p_{f}}$ is the full subcategory of fibrant objects. But in $\mathscr{S}_{p} X$ is fibrant if and only if $P s_{k}(X)$ is fibrant for every integer $k$, if and only if $P s_{k}(X)$ is a Kan set for every $k$. Thus $\mathscr{S p}_{f}=\mathscr{S}_{p_{E}}$, and in this category a morphism $f$ is a weak equivalence if and only if $P_{S_{k}}(f)$ is a weak equivalence for every integer $k$ by [3, Prop. 9.2]. Thus the two definitions of weak equivalences coincide in $\mathscr{S} p_{E}$. On the other hand $H o \mathscr{S}_{p_{E}} \approx\left(\mathscr{S}_{p_{E}}\right)_{h}$ by [4, Theorem 4.12].

Remark 2.12. In [1] and [2] Hastings claims that $f: X \rightarrow Y$ is a weak equivalence (in the classical sense) if and only if $P s_{k}(f)$ is a weak equivalence for every $k$. This of course is valid for Kan spectra, but not in general. Take for instance the sphere spectrum $\Gamma \dot{\Delta}(n)$. By [3, Prop. 5.3] the map $\pi_{*} \Gamma \dot{\Delta}(n) \rightarrow \pi_{*} F \Gamma \dot{\Delta}(n)$ is an isomorphism. Because $F \Gamma \dot{\Delta}(n)$ is a Kan spectrum by [3, Example 7.5], $\pi_{k} F \Gamma \dot{\Delta}(n)=$ $=\pi_{k+t} P s_{t} F \Gamma \dot{\Delta}(n)$ for every $t$ such that $k+t \geqq 0$. If the result mentioned above held we would have

$$
\operatorname{colim}_{t} \pi_{k+t} S^{t} \dot{\Delta}(n)=\operatorname{colim}_{t} \pi_{k+t} P S_{t} \Gamma \dot{\Delta}(n)=\pi_{k+t} P s_{t} \Gamma \dot{\Delta}(n)=\pi_{k+t} S^{t} \dot{\Delta}(n)
$$

for every $t$ such that $k+t \geqq 0$. But this would not seem to hold; choose for instance $n=1, k=3, t=3$. Of course the error lies in the fact that a theorem, [2, Prop 4.7], proved only for Kan sets is applied to the general case ([2, Prop. 4.8]). 


\title{
References
}

[1] Edwards, D. A., and H. M. Hastings: Čech and Steenrod homotopy theories with applications to geometric topology. - Lecture Notes in Mathematics 542, Springer-Verlag, BerlinHeidelberg-New York, 1976, 1-296.

[2] Hastings, H. M.: On the homotopy theory of simplicial spectra. - State University of New York at Binghamton, Binghamton, 1974, 1-22.

[3] KAN, D. M.: Semisimplicial spectra. - Illinois J. Math. 7, 1963, 463-478.

[4] Malm, K.: On the stabilization of 2-categories. - Ann. Acad. Sci. Fenn. Ser. A I Diss. 3, 1975, $1-42$.

[5] Quillen, D. G.: Homotopical algebra. - Lecture Notes in Mathematics 43, Springer-Verlag, Berlin-Heidelberg-New York, 1967.

[6] Quillen, D. G.: Rational homotopy theory. - Ann. of Math. 90, 1969, 205-295.

[7] Tierney, M.: Categorical constructions in stable homotopy theory. - Lecture Notes in Mathematics 87, Springer-Verlag, Berlin-Heidelberg-New York, 1969, 1—65.

\author{
University of Helsinki \\ Department of Mathematics \\ SF-00100 Helsinki 10 \\ Finland
}

Received 1 August 1977 